\title{
Characterization and Fungicide Sensitivity of Colletotrichum Species Causing Strawberry Anthracnose in Eastern China
}

\author{
Liqing Zhang, ${ }^{1,2}$ Lili Song, ${ }^{3}$ Xiangming Xu, ${ }^{4}$ Xiaohua Zou, ${ }^{1}$ Ke Duan, ${ }^{1,3, \dagger}$ and Qinghua Gao ${ }^{1, \dagger}$ \\ ${ }^{1}$ Shanghai Key Laboratory of Protected Horticultural Technology, Forestry and Fruit Tree Research Institute, Shanghai Academy \\ of Agricultural Sciences, Shanghai 201403, China \\ ${ }^{2}$ Shanghai Agricultural Seed \& Seedling Co., Ltd. \\ ${ }^{3}$ College of Food Science, Shanghai Ocean University, Shanghai 201306, China \\ ${ }^{4}$ NIAB EMR, New Road, East Malling, Kent ME19 6BJ, U.K.
}

\begin{abstract}
Strawberry anthracnose caused by Colletotrichum spp. is one of the most serious diseases in the strawberry fields of China. In total, 196 isolates of Colletotrichum were obtained from leaves, stolons, and crowns of strawberry plants with anthracnose symptoms in eastern China and were characterized based on morphology, internal transcribed spacer (ITS), and $\beta$-tubulin (TUB2) gene sequences. All 196 isolates were identified as the Colletotrichum gloeosporioides species complex. In total, 62 strains were further identified at the species level by phylogenetic analyses of multilocus sequences of ITS, glyceraldehyde-3-phosphate dehydrogenase (GAPDH), actin (ACT), Apn2-Mat1-2 intergenic spacer and partial mating type (ApMat), calmodulin (CAL), and TUB2. Three species from

than the other two species when grown above $36^{\circ} \mathrm{C}$. The inoculation of strawberry plants confirmed the pathogenicity of all three species. $C$. siamense isolates resulted in the highest disease severity. The in vitro sensitivities of $C$. siamense and $C$. fructicola isolates to azoxystrobin and three demethylation-inhibitor (DMI) fungicides (difenoconazole, tebuconazole, and prochloraz) were determined. Both species were sensitive to DMI fungicides but not to azoxystrobin. $C$. siamense isolates were more sensitive to prochloraz, while $C$. fructicola isolates were more sensitive to difenoconazole and tebuconazole. The present study provides valuable information for the effective management of strawberry anthracnose.
\end{abstract} the $C$. gloeosporioides species complex were identified: Colletotrichum siamense, $C$. fructicola, and C. aenigma. Isolates of $C$. siamense were tolerant to high temperatures, with a significantly larger colony diameter
Keywords: anthracnose, Colletotrichum gloeosporioides, fungicide sensitivity, strawberry
Strawberry (Fragaria $\times$ ananassa) is a popular and widely consumed fruit (Khoshnevisan et al. 2013, 2014). Strawberry production has expanded rapidly in recent years, and China has become one of the world's largest strawberry producers and exporters. In 2016, the global production of strawberry fruit was 9.2 million tons, with China accounting for $41 \%$ of the total (FAOSTAT 2016). With the increase in production and expansion of land allocated to strawberry growth, fungal diseases have become a major factor influencing strawberry yield and quality in China.

Anthracnose, caused by Colletotrichum spp., is a major strawberry disease in subtropical climates, in which hot and humid conditions favor disease development and can result in significant crop losses (Anciro et al. 2018). Strawberry anthracnose symptoms occur on all plant parts (Buddie et al. 1999). Lesions on stolons and petioles, as well as irregular leaf spots, are mainly found in plant nurseries (Freeman et al. 2001). In field crops, flower blight and fruit rot are common symptoms (Howard et al. 1992). Crown rot is characterized

†Corresponding authors: Q. Gao; qhgao20338@sina.com; and K. Duan; kduan936@126.com

L. Zhang and L. Song contributed equally to this work.

Funding: This work was financially supported by the Shanghai Rising-Star Program (18QB1402800), The Science and Technology Commission of Shanghai Municipality (key program 16391901400), and Shanghai Municipal Agricultural Commission Project (HuNong Ke Zhong Zi-2017-2-1).

*The $\boldsymbol{e}$-Xtra logo stands for "electronic extra" and indicates that one supplementary table is published online.

The author(s) declare no conflict of interest.

Accepted for publication 3 February 2020.

(C) 2020 The American Phytopathological Society by reddish-brown necrotic areas (Cai et al. 2009, 2011) and, in some cases, stunting and chlorosis have been associated with root necrosis (Freeman et al. 2001).

Colletotrichum is an important genera of plant-pathogenic fungi (Damm et al. 2012; Udayanga et al. 2013). The internal transcribed spacer (ITS) sequences alone, which have long been used to classify fungal species, cannot fully differentiate Colletotrichum spp. (Weir et al. 2012). A multilocus phylogeny coupled with morphological data are now usually used for the identification of Colletotrichum spp. (Cai et al. 2009, 2011). Three species have been reported as causal agents of strawberry anthracnose: Colletotrichum acutatum, C. fragariae (which is now known as $C$. theobromicola), and $C$. gloeosporioides (Denoyes-Rothan et al. 2003). Based on multilocus phylogeny and morphological data, C. acutatum and C. gloeosporioides have been defined as species complexes, containing 36 and 38 species, respectively (Damm et al. 2012; Marin-Felix et al. 2017; Weir et al. 2012).

C. gloeosporioides was described for the first time as a strawberry pathogen in the southeastern United States in 1930 (Brooks 1931). In the United States, epidemics of strawberry crown rot are mainly caused by $C$. gloeosporioides (Ureña-Padilla et al. 2002) but $C$. gloeosporioides is found only occasionally on strawberry in Europe (Freeman and Katan 1997; Van Hemelrijck et al. 2010). In Asia, C. gloeosporioides is the main cause of strawberry anthracnose. In Japan, Korea (Nam et al. 2013; Suzuki et al. 2010), and China (Jayawardena et al. 2016), C. fructicola is the predominant species.

Strawberry anthracnose has been mainly managed with fungicide applications. Since the 1990s, the most commonly used fungicides are benzimidazole fungicides such as carbendazim and thiophanate methyl (Han et al. 2009). However, since 2009 in China, benzimidazole-resistant $C$. gloeosporioides isolates have been frequently found in strawberry, leading to disease control failures (Han et al. 2009). At present, there are no other types of fungicides registered for controlling strawberry anthracnose in China $\mathrm{Xu}$ et al. 2014). Demethylation-inhibitor (DMI) fungicides are alternatives to benzimidazole fungicides, and some of them (prochloraz 
and tebuconazole) have been registered for use against grape anthracnose. The baseline sensitivity levels of $C$. gloeosporioides isolates to prochloraz and tebuconazole in strawberry and grape have been determined (Xu et al. 2014). Quinone outside inhibitor (QoI) fungicides are the primary choice for anthracnose control caused by Colletotrichum spp. in commercial strawberry fields in the United States (Brannen and Smith 2015).

Strawberry anthracnose was first reported in eastern China in 1990 (Hu 1990). Recent studies in some areas of China have identified the Colletotrichum spp. causing strawberry anthracnose to be C. nymphaeae (C. acutatum species complex) (Hubei Province and Beijing City), C. fructicola (Hubei, Liaoning, and Hainan Provinces and Beijing City), C. gloeosporioides (Hubei Province), C. aenigma (Hubei Province), and C. changpingense (C. gloeosporioides species complex) (Beijing City) (Han et al. 2016; Jayawardena et al. 2016). Strawberry is among the most widely grown fruit in eastern China, which has a moderate climate with plentiful rainfall (Wang et al. 2012). There have not been systematic studies of strawberry anthracnose in eastern China. The objectives of the present study were to (i) identify the Colletotrichum spp. associated with strawberry anthracnose in eastern China based on multilocus phylogeny and morphology, (ii) determine the pathogenicity of different Colletotrichum spp., and (iii) determine the sensitivity of Colletotrichum spp. to QoI (azoxystrobin) and DMI fungicides.

\section{Materials and Methods}

Fungal isolate collection. From 2016 to 2018, leaves, stolons, and crowns of strawberry plants with anthracnose symptoms were collected from six provinces of eastern China (Fig. 1). Colletotrichum spp. were isolated as described (Cao et al. 2019). Three pieces ( 5 by $5 \mathrm{~mm}$ ) of tissue were taken from the margins between necrotic and apparently healthy tissues of each plant and surface sterilized in $70 \%$ ethanol for $30 \mathrm{~s}$ and then $1 \% \mathrm{NaClO}_{3}$ for $1 \mathrm{~min}$. Finally, the samples were rinsed three times with sterilized distilled water and dried before being plated onto potato dextrose agar (PDA). PDA plates were incubated at $28^{\circ} \mathrm{C}$ under continuous darkness for 3 to 7 days. Single-spore cultures were obtained for each isolate in accordance with a previously published method (Cai et al. 2009), and then they were subcultured onto fresh PDA plates.
DNA extraction, PCR amplification, and DNA sequencing. Isolates were grown on PDA at $28^{\circ} \mathrm{C}$ for 7 days. Mycelia of each isolate were scraped from the colony surface with a sterile $20-\mu$ l pipette tip. Total genomic DNA was extracted using the E.Z.N.A. Fungal DNA Mini Kit (Omega Bio-tek, Germany) according to the manufacturer's instructions.

As an initial analysis of genetic diversity, ITS and the $\beta$-tubulin (TUB2) gene were amplified from all of the isolates obtained in this study using the primers ITS1/ITS4 and T1/Bt2b, respectively (Glass and Donaldson 1995; O'Donnell and Cigelnik 1997; White et al. 1990).

A subset of 62 isolates, representing different geographic origins and genetic diversity (from initial ITS and TUB2 analysis), was then selected for further investigation. The ITS and partial sequences of the glyceraldehyde-3-phosphate dehydrogenase (GAPDH), actin (ACT), calmodulin (CAL), TUB2, and the Apn2-Mat1-2 intergenic spacer and partial mating type (Mat1-2) gene (ApMat) were amplified and sequenced using the primer pairs ITS1/ITS4 (White et al. 1990), GDF1/GDR1 (Templeton et al. 1992), ACT512F/ACT783R (Carbone and Kohn 1999), CL1/CL2A (O'Donnell et al. 2000), T1/Bt2b (Glass and Donaldson 1995; O'Donnell and Cigelnik 1997), and AMF1/AMR1 (Silva et al. 2012), respectively .

The PCR products were examined by electrophoresis in $1.5 \%$ agarose gels stained with 4S Red Plus Nucleic Acid Stain (Sangon Biotech Co., Ltd., China) in a 1.0× Tris-acetate acid-EDTA buffer and photographed under UV light. The PCR amplifications were performed with a Mastercycler nexus (Eppendorf, Germany) in a $50-\mu l$ reaction volume. The PCR mixtures contained $1 \mu$ l of DNA template, $25 \mu \mathrm{l}$ of TaKaRa Premix Taq (Ex Taq version; TaKaRa, Tokyo, Japan), and $1.5 \mu \mathrm{M}$ each primer. The PCR amplification protocols were performed as described by Cao et al. (2019) but the denaturing temperatures were adjusted to $55^{\circ} \mathrm{C}$ for ITS and $C A L, 58^{\circ} \mathrm{C}$ for $G A P D H, 60^{\circ} \mathrm{C}$ for $A C T$ and $T U B 2$, and $61^{\circ} \mathrm{C}$ for ApMat. The PCR products were purified using the AxyPrep PCR Cleanup Kit (Axygen Biosciences, China) following the manufacturer's instructions. Sequencing the PCR amplicons was carried out by Sangon Biotech Co., Ltd.

Phylogenetic analyses. Multiple sequence alignments incorporating each gene were performed with BioEdit (Hall 1999) and ClustalX (Thompson et al. 2003). Alignments were manually adjusted to allow

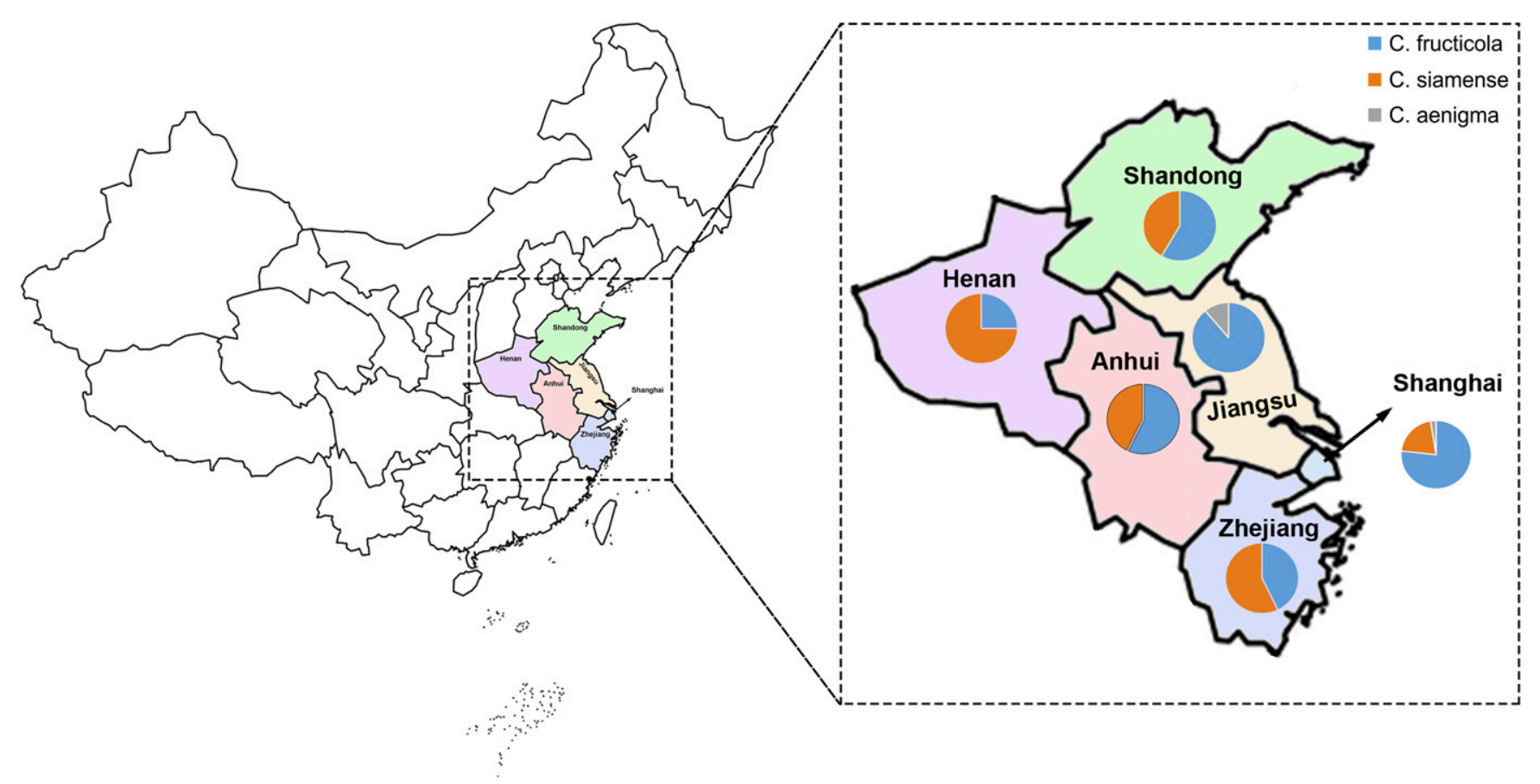

Fig. 1. Map showing locations in eastern China where strawberry plants were sampled for Colletotrichum spp. 
for the maximum alignment and to minimize gaps. The consensus sequences were deposited in GenBank (Supplementary Table S1). The partial DNA sequences of ITS, GAPDH, ACT, ApMat, CAL, and TUB2 for 62 representative isolates of the $C$. gloeosporioides complex from different regions, 54 reference isolates of $C$. gloeosporioides, and the isolate MAFF305972 of $C$. boninense were used to establish a sixlocus combined dataset $(A C T+A p M a t+C A L+G A P D H+\mathrm{ITS}+T U B 2)$ for the Bayesian inference analysis. The latter (MAFF305972) was used as an outgroup. The phylogenetic analysis was conducted using a Markov Chain Monte Carlo algorithm to generate trees with Bayesian posterior probabilities in MrBayes v.3.2.2 (Ronquist et al. 2012). MrModeltest v.2.3 (Nylander 2004) was used to carry out the statistical selection of best-fit models of nucleotide substitutions using the corrected Akaike information criteria. Specifically, the models selected were GTR+I for $A C T$ and ITS, K80+G for ApMat, GTR+I+G for $C A L, \mathrm{HKY}$ for $G A P D H$, and $\mathrm{SYM}+\mathrm{G}$ for $T U B 2$; these models were incorporated into the analysis. The heating parameter was set to 0.2 , and analyses were stopped once the average standard deviations of the split frequencies were below 0.01. Using these criteria, the first $25 \%$ of generations were discarded as burn-in. The generated $50 \%$ majority rule consensus tree was visualized using FigTree, version 1.4.2 (http://tree.bio.ed.ac.uk/software/figtree/). Clades were regarded as significantly supported if they had a posterior probability $\geq 0.95$ (Ronquist et al. 2012).

Morphological analysis. Mycelial discs ( $9 \mathrm{~mm}$ in diameter) were taken from 7-day-old cultures, plated on PDA, and incubated at $28^{\circ} \mathrm{C}$ in the dark. The colony diameter (in millimeters) was measured in two perpendicular directions. The daily growth rate was calculated as the 7-day average of mean daily growth (millimeters per day), and colony characteristics were recorded. The experiment was designed as a randomized complete block. There were three replications of each isolate per temperature treatment. Characteristics of conidia, conidiophores, and appressoria were determined using methods described previously (Cai et al. 2009) and assessed using a digital microscope (DM2500; Leica, Germany). Appressoria induced on a synthetic nutrient-poor agar medium were measured using a slide culture technique (Cai et al. 2009).

Effects of temperature on mycelial growth. Based on the phylogenetic analyses, 13 Colletotrichum isolates obtained from different locations were evaluated to determine the effects of temperatures on radial colony growth in culture. The selected isolates consisted of five C. siamense isolates (GQHSD5, GQHHN12, GQHAH2, GQHZJ15, and GQH12), five C. fructicola isolates (GQHSD2, GQHZJ1, GQHAH6, GQHHN8, and GQH131), and three $C$. aenigma isolates (GQH124, GQH116, and GQHJS18). The protocols

Table 1. Rating scale for leaves and petioles inoculated by Colletotrichum spp.

\begin{tabular}{lcc}
\hline Rating & Area of leaf surface $(\%)$ & Length of lesions (petioles) $(\mathbf{m m})$ \\
\hline 0 & Healthy leaves with no lesions & Healthy petioles with no lesions \\
1 & $0.1-5.0$ & $0.1-2$ \\
3 & $5.1-15.0$ & $2.1-5.0$ \\
5 & $15.1-30.0$ & $5.1-9.0$ \\
7 & $30.1-50.0$ & $9.1-15.0$ \\
9 & $50.1-100$ or dead leaves & $>15.0$ or leaf wilting \\
\hline
\end{tabular}

performed were as described by Han et al. (2016). The plates were wrapped with parafilm and placed in an incubator (MJP-250; Shanghai Jing Hong Laboratory Instrument Co., Ltd., China) in the dark with the temperature set at one of the following values: 5, 10, $15,20,25,28,30,32,34,36$, or $38^{\circ} \mathrm{C}$. After 5 days, the colony diameter (in millimeters) was measured and the daily growth rate was calculated. The experiment was designed as a randomized complete block. There were three replications of each isolate per temperature treatment.

Pathogenicity assay. Strawberry plants (cultivar Benihoppe) were used to confirm the pathogenicity of identified Colletotrichum spp. Three isolates of each identified species were chosen for pathogenicity tests: $C$. siamense (GQH12, GQHZJ15, and GQHAH8), $C$. fructicola (GQH26, GQHHN8, and GQHAH6), and C. aenigma (GQH124, GQHJS18, and GQH116). To further investigate the pathogenicity of the $C$. gloeosporioides species complex, 10 isolates of $C$. siamense (GQHSD5, GQH12, GQH39, GQH46, GQH137, GQHAH2, GQHAH8, GQHHN12, GQHZJ15, and GQHZJ19) and 10 isolates of $C$. fructicola (GQHSD2, GQH26, GQH64, GQH94, GQH131, GQH133, GQHJS19, GQHAH6, GQHHN8, and GQHZJ1) were chosen for pathogenicity tests. Conidial suspensions were prepared from 7-day-old cultures as previously described (Han et al. 2016). Stolon-derived healthy plants with more than 10 fully expanded compound leaves were inoculated by spraying a conidial suspension $\left(10^{6}\right.$ conidia/ml with $0.01 \%$ [vol/vol] Tween 20 in sterile water) until runoff occurred (Freeman and Katan 1997). Mock inoculations of plants were carried out using just the Tween $20-$ water solution. Six plants, one per pot, for each isolate were inoculated, covered with plastic bags, and immediately placed in growth chambers at 90 to $100 \%$ relative humidity at $25^{\circ} \mathrm{C}$ in the dark for $48 \mathrm{~h}$. The plastic bags were then removed and plants were maintained in growth chambers with a 12-h photoperiod at 25 and $20^{\circ} \mathrm{C}$ (day and night, respectively). Disease development was recorded 5 to 10 days after inoculation, as previously described (Han et al. 2016). The rating scale for leaves and petioles is listed in Table 1. An index of the disease was also calculated for each isolate, using Horiuchi and Hori's method: index of disease $(\%)=\left[\left(n_{0} \times 0+n_{1} \times 1+n_{3} \times 3+n_{5} \times 5+n_{7} \times 7+n_{9} \times 9\right) /\right.$ $9 N] \times 100$, where $n$ represents the number of leaves or petioles in each class $(0,1,3,5,7$, and 9$)$ and $N$ represents the total number of leaves or petioles (Horiuchi and Hori 1980). These experiments were performed twice.

Fungicide sensitivity assays. In total, 15 isolates from $C$. siamense (GQHSD5, GQH17, GQH12, GQH39, GQH46, GQH137, GQHAH2, GQHAH8, GQHAH11, GQHHN11, GQHHN12, GQHHN13, GQHZJ15, GQHZJ19, and GQHZJ20) and 15 isolates from C. fructicola (GQHSD2, GQHSD7, GQH26, GQH64, GQH94, GQH131, GQH133, GQHAH6, GQHAH16, GQHHN8, GQHZJ1, GQHJS1, GQHJS3, GQHJS19, and GQHJS21) were selected based on geographical origin and species, and they were tested for their sensitivities. Each chemical was prepared separately in sterile distilled water and adjusted to a concentration of $10,000 \mu \mathrm{g} / \mathrm{ml}$ as the stock solution. The stock solutions were diluted to a series of concentrations and added to cooling, melted PDA (approximately $50^{\circ} \mathrm{C}$ ) after autoclaving (Table 2). Mycelial plugs $(5 \mathrm{~mm}$ in diameter) were removed from the margins of colonies of 7-day-old cultures actively growing on PDA and placed

Table 2. List of the fungicides used in this study ${ }^{z}$

\begin{tabular}{lllr}
\hline FRAC code & Chemical name & Manufacturer (in China) & Concentration (a.i. $\boldsymbol{\mu g} / \mathbf{m l})$ \\
\hline 11 & Azoxystrobin & 25SC; Syngenta Nantong Crop Protection Co. & $0,100,250,500,1,000,2,000$ \\
3 & Ltd. & 10WDG; Syngenta Nantong Crop Protection & $0,0.01,0.1,2.5,5,10$ \\
3 & Co. Ltd. & $0,0.01,0.05,0.1,10,20$ \\
3 & Tebuconazole & 43SC; Bayer Crop Sciences Co. Ltd. & $0,0.01,0.05,0.1,0.5,1$ \\
& Prochloraz & 45ME; Shenzhen Noposion Agrochemicals & \\
\hline
\end{tabular}

${ }^{\mathrm{z}}$ FRAC codes of fungicides according to the Fungicide Resistance Action Committee (FRAC) website (https://www.frac.info); a.i. = active ingredient. 


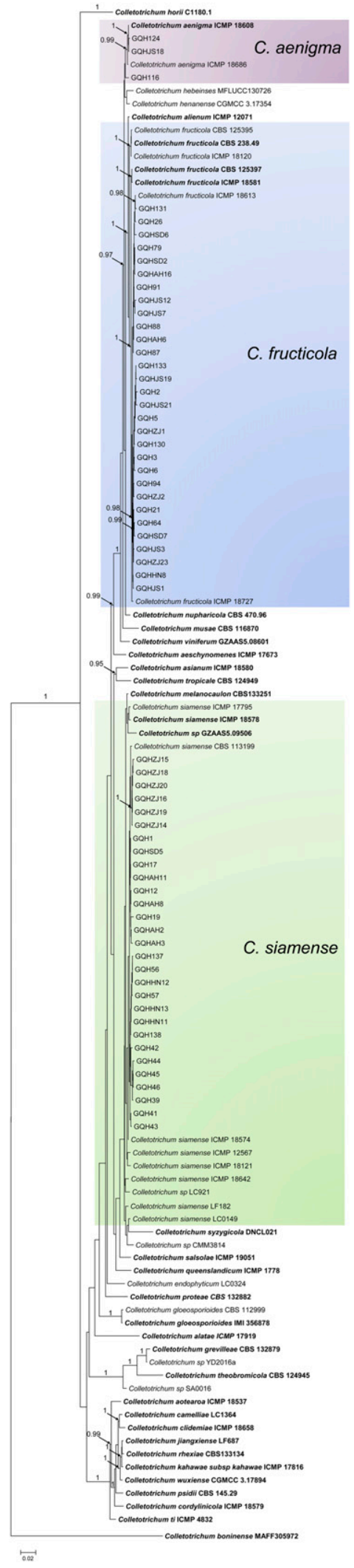

Fig. 2. Phylogenetic consensus tree based on Bayesian inference, illustrating the relationships within the Colletotrichum gloeosporioides species complex of isolates obtained from strawberry plants in eastern China. The tree was built with a Bayesian analysis of concatenated sequences of actin (ACT), Apn2-Mat1-2 intergenic spacer and partial mating type (ApMat), calmodulin (CAL), glyceraldehyde-3-phosphate dehydrogenase (GAPDH), internal transcribed spacer (ITS), and $\beta$-tubulin (TUB2). The Markov chain was run for 5,000,000 generations, each locus having a separate model of DNA evolution. C. boninense (MAFF305972) was used as the outgroup. Bayesian posterior probability values $\geq 0.95$ are shown next to the nodes. onto the center of plates containing fungicide. Plates were incubated at $28^{\circ} \mathrm{C}$ in the dark. The plates were incubated for 7 days at $28^{\circ} \mathrm{C}$, and the diameter of each colony was measured in two perpendicular directions. There were three replicate plates, and the experiments were repeated twice.

The fungicide concentration at which fungal development is inhibited by $50 \%\left(\mathrm{EC}_{50}\right)$ was used to measure fungicide sensitivity (Wong and Midland 2007; Wong and Wilcox 2002). The fungal relative growth (RG) at a given fungicide concentration was calculated as a percentage of fungal growth of the control plates. The $\mathrm{EC}_{50}$ value was estimated by the linear regression of the probit-transformed relative inhibition value $(1-\mathrm{RG})$ at the $\log 10$ transformed-fungicide concentration. The $\mathrm{EC}_{50}$ value for each isolate was calculated as the mean of the three experiments, and the correlation coefficients ( $r$ ) among $\mathrm{EC}_{50}$ values of different fungicides were determined using the statistical algorithms of SAS software (version 9.4 for Windows; SAS Institute, Cary, NC, U.S.A.).

Data analyses. Daily growth rate data were subjected to an analysis of variance (ANOVA) followed by Duncan's multiple range test using SAS software. The minimum, optimum, and maximum growth temperature data were analyzed using the general linear model procedure in SAS. Species were treated as fixed effects and isolates were
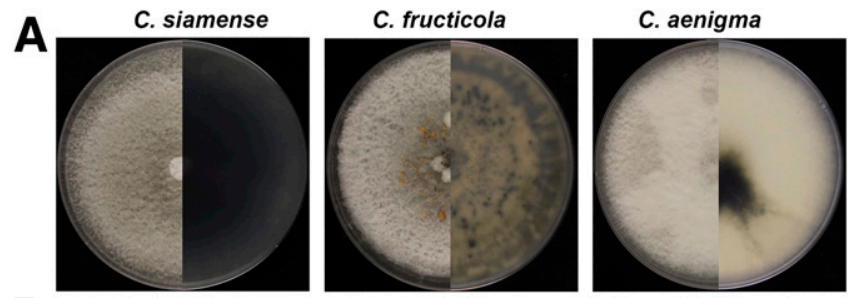

B
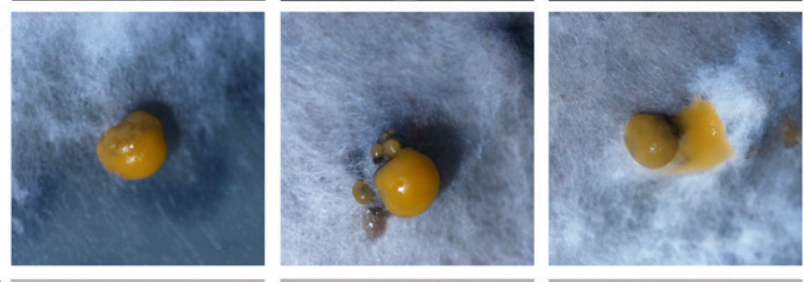

C
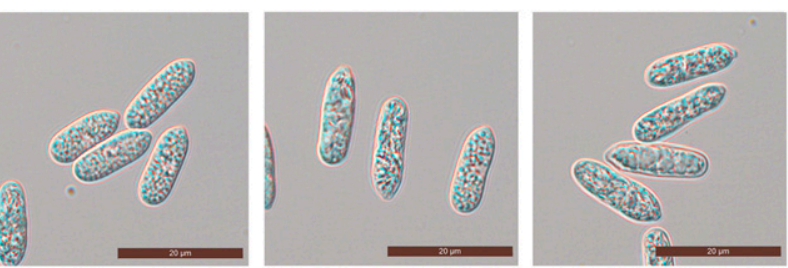

D
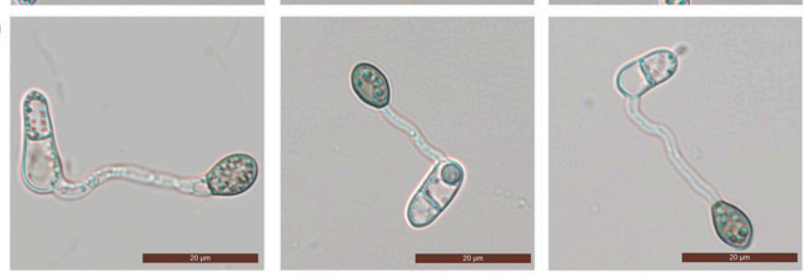

E
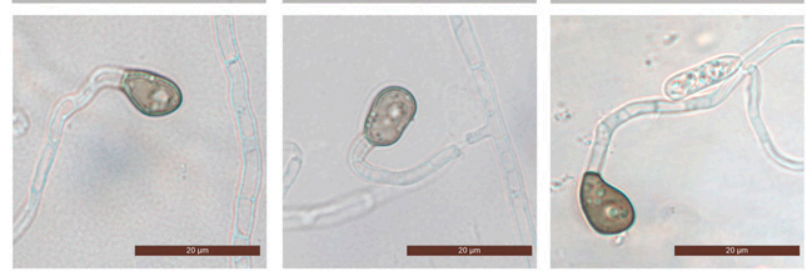

Fig. 3. Culture characteristics and microscopic features of the Colletotrichum gloeosporioides species complex from strawberry anthracnose. A, Upper and reverse view of 1-week-old colonies on potato dextrose agar; $\mathbf{B}$, conidiomata of the C. gloeosporioides species complex; $\mathbf{C}$, micrographs of conidia of the $\mathrm{C}$. gloeosporioides species complex; $\mathrm{D}$, micrographs of mycelial appressoria of the $\mathrm{C}$. gloeosporioides species complex; and $\mathrm{E}$, micrographs of conidial appressoria of the C. gloeosporioides species complex conidia. Scale bars $=20 \mu \mathrm{m}$. 
treated as random effects. When $F$ values were significant $(P<0.05)$, the least significant difference tests were used to compare the means of three species. Data from the pathogenicity assay were subjected to an ANOVA using SAS software to determine the significance levels of treatment differences. Means for different species from the pathogenicity test were separated using least significant difference tests $(P=0.05)$. Disease indices for $C$. siamense $(n=10)$ and $C$. fructicola $(n=10)$ isolates on strawberry plants were analyzed using Duncan's multiple range test. Mean $\log \mathrm{EC}_{50}$ values for $C$. siamense and $C$. siamense for the same fungicide were compared using two-tailed $t$ tests with $\alpha=0.05$. An ANOVA was performed on the $\log \mathrm{EC}_{50}$ values to compare the sensitivity of each species to the fungicides tested when the fungicide and isolate were treated as factors. To determine levels of cross-sensitivity between pairs of tested fungicides, coefficients of correlation using the $\log \mathrm{EC}_{50}$ values between any two fungicides were calculated for each species.

\section{Results}

Molecular identification and phylogenetic analyses. In total, 196 Colletotrichum strains were isolated from diseased strawberry plant samples (leaves, stolons, and crowns) from six provinces (Shanghai, Anhui, Henan, Shandong, Jiangsu, and Zhejiang) of eastern China (Fig. 1). Based on the phylogenetic analysis of the combined ITS and TUB2 alignment and the colony morphological characteristics on PDA, all of these Colletotrichum isolates were initially identified as the C. gloeosporioides species complex. In total, 62 isolates were subsequently selected for further analyses on the basis of their origins, colony characteristics, and conidial morphologies (Supplementary Table S1).

Multilocus phylogenetic analysis. The six-locus phylogenetic analysis of the C. gloeosporioides species complex included 54 reference isolates and $C$. boninense (MAFF305972) as the outgroup (Fig. 2). The Bayesian inference was performed with the combined ACT, ApMat, CAL, GAPDH, ITS, and TUB2 sequences, and comprised 2,916 characters after alignment. The boundaries of the loci used in the alignment were as follows: ACT, 1 to 226; ApMat, 227 to 1,$011 ; C A L, 1,012$ to 1,$711 ; G A P D H, 1,712$ to 1,946 ; ITS, 1,947 to 2,412; and $T U B 2,2,413$ to 2,916. The 62 tested isolates from the $C$. gloeosporioides species complex clustered in three clades: 29 isolates clustered with $C$. siamense, 30 isolates clustered with $C$. fructicola, and 3 isolates clustered with C. aenigma (Fig. 2).

Morphological and cultural characteristics. Distinct colony morphologies were observed for each species after 7 days on PDA. Isolates of $C$. siamense produced gray colonies with sparse, gray aerial mycelia. The reverse sides of the colonies were gray to black. Isolates of $C$. fructicola varied from gray to dark gray on PDA, with dense gray aerial mycelia and a few bright orange conidial masses near the inoculum point. Isolates of $C$. aenigma produced white colonies with dense gray to white aerial mycelia. The reverse sides of the colonies were pale orange toward the centers. Few differences in conidial morphology were observed among the three species. All three species had long elliptical conidia with obtuse to slightly rounded ends (Fig. 3). The growth rate of the $C$. siamense isolates was more rapid than those of $C$. aenigma and $C$. fructicola $(P=0.0203)$ (Table 3$)$.
Effects of temperature on mycelial growth. The optimum temperature for all three species was $28^{\circ} \mathrm{C}$. The tolerance levels to high temperatures were similar for $C$. fructicola and $C$. aenigma. For $C$. fructicola and $C$. aenigma isolates, no mycelial growth was observed at temperatures of $36^{\circ} \mathrm{C}$ and above. Isolates of $C$. siamense were tolerant to high temperatures, resulting in significantly larger colony diameters than the other two species when the temperature was $>36^{\circ} \mathrm{C}(P<0.05)$. Mycelial growth of $C$. siamense in vitro was observed at $38^{\circ} \mathrm{C}$, with an average growth rate of $0.35 \mathrm{~mm} /$ day (Table 3; Fig. 4).

Pathogenicity tests. The pathogenicity levels of the Colletotrichum isolates were determined on both the leaves and petioles of strawberry plants. Necrotic spots were visible 3 days after inoculation. Disease symptoms were observed on petioles 7 days after inoculation. Disease severity caused by the three species was significantly different. Isolates of $C$. siamense showed the greatest disease severity $(P<0.05)$. The disease indices of the leaves and petioles were 91.3 and 83.7, respectively. C. fructicola species also showed strong pathogenicity on both the leaves and petioles of strawberry plants (Table 4). Plant mortalities caused by $C$. siamense and $C$. fructicola at 12 days postinoculation (dpi) were 60.0 and $51.7 \%$, respectively. C. aenigma-infected leaves and petioles showed lower mean infection incidences ( 68.4 and $47.4 \%$, respectively). For $C$. siamense, the disease development resulting from most isolates, except for GQH46 and GQHAH2, was similar. The disease index of GQH46 was significantly higher than those of the other isolates, especially at 4 and 6 dpi. In contrast, the disease index of GQHAH2 was significantly lower than those of the other tested isolates at almost all time points (Fig. 5A). For C. fructicola, compared with the other isolates, GQHSD2 was the most virulent on strawberry leaves, especially at 8 ,

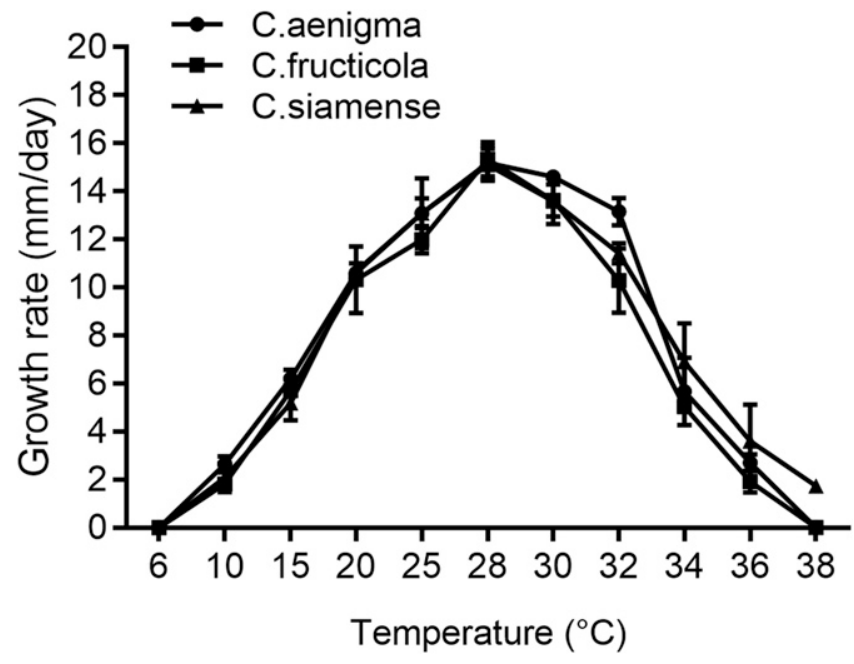

Fig. 4. Colony growth rate of the Colletotrichum gloeosporioides species complex from strawberry plants under different temperatures.

Table 3. Morphological characteristics of Colletotrichum gloeosporioides complex causing anthracnose of strawberry plants in eastern China

\begin{tabular}{|c|c|c|c|c|c|c|c|c|c|}
\hline \multirow[b]{3}{*}{ Species } & \multirow[b]{3}{*}{ Colony ${ }^{\mathbf{y}}$} & \multicolumn{5}{|c|}{ Conidial morphology $y^{x}$} & \multirow[b]{3}{*}{ Growth $^{\mathrm{Z}}$} & \multirow{2}{*}{\multicolumn{2}{|c|}{ Characteristics of appressoria }} \\
\hline & & \multicolumn{2}{|c|}{ Length $(\mu \mathrm{m})$} & \multicolumn{2}{|c|}{ Width $(\mu \mathrm{m})$} & \multirow[b]{2}{*}{ Shape } & & & \\
\hline & & Range & Mean & Range & Mean & & & Conidial & Mycelial \\
\hline C. siamense & $\begin{array}{l}\text { White to pale gray, } \\
\text { dark gray }\end{array}$ & $13.9-19.1$ & $16.5 \pm 0.5$ & $4.7-6.5$ & $5.4 \pm 0.2$ & Long elliptical & $6.84 \pm 0.14 \mathrm{a}$ & $\begin{array}{l}\text { Brown to dark black, spindle to } \\
\text { slightly irregular }\end{array}$ & $\begin{array}{l}\text { Light brown to brown, spindle } \\
\text { or irregular }\end{array}$ \\
\hline C. aenigma & White to taupe & $11.4-17.5$ & $14.6 \pm 0.7$ & $4.7-5.9$ & $5.3 \pm 0.4$ & Long elliptical & $6.59 \pm 0.16 b$ & $\begin{array}{l}\text { Brown to dark black, spindle to } \\
\text { slightly irregular }\end{array}$ & $\begin{array}{l}\text { Light brown to brown, spindle } \\
\text { or irregular }\end{array}$ \\
\hline C. fructicola & $\begin{array}{l}\text { White to } \\
\text { black/green }\end{array}$ & $13.4-18.3$ & $15.8 \pm 0.4$ & $4.2-5.5$ & $4.9 \pm 0.6$ & Long elliptical & $6.43 \pm 0.25 b$ & $\begin{array}{l}\text { Brown to dark black, spindle to } \\
\text { slightly irregular }\end{array}$ & $\begin{array}{l}\text { Light brown to brown, spindle } \\
\text { or irregular }\end{array}$ \\
\hline
\end{tabular}

${ }^{\mathrm{x}}$ Data are mean \pm standard deviation; range is approximate.

y Colony morphology.

${ }^{\mathrm{z}}$ Growth rate $\left(\mathrm{mm} \mathrm{day}^{-1}\right)$. Means followed by the same letter were not significantly different at $P \leq 0.05$. 
10, and 12 dpi. GQHZJ1 and GQH133 showed the lowest disease indices at almost all time points (Fig. 5B).

Sensitivity to fungicides. Isolates of $C$. siamense and $C$. fructicola, the main species causing strawberry anthracnose in this study, were chosen for the determination of sensitivities to fungicides using the mycelial growth method. For $C$. siamense isolates, the $\mathrm{EC}_{50}$ value (mean \pm standard deviation) was $0.056 \pm 0.030 \mu \mathrm{g} / \mathrm{ml}$ for

Table 4. Pathogenicity analyses of Colletotrichum gloeosporioides complex isolates from strawberry plants ${ }^{\mathrm{y}}$

\begin{tabular}{lllc}
\hline & \multicolumn{3}{c}{ Disease index (12 days after inoculation) } \\
\cline { 2 - 4 } Species & Foliar & Petiole & Mortality $(\%)$ \\
\hline C. siamense & $91.3 \mathrm{a}$ & $83.7 \mathrm{a}$ & $60.0 \mathrm{a}$ \\
C. fructicola & $84.9 \mathrm{a}$ & $70.0 \mathrm{ab}$ & $51.7 \mathrm{a}$ \\
C. aenigma & $68.4 \mathrm{~b}$ & $47.4 \mathrm{~b}$ & $30.0 \mathrm{~b}$ \\
\hline
\end{tabular}

y Data are mean values. Mean values with the same letters were not statistically different $(P>0.05)$ according to the least significant difference test.

z Percentages of plant mortality were evaluated at 12 days after inoculation. difenoconazole, $8.885 \pm 0.53 \mu \mathrm{g} / \mathrm{ml}$ for tebuconazole, $0.122 \pm$ $0.070 \mu \mathrm{g} / \mathrm{ml}$ for prochloraz, and $>100 \mu \mathrm{g} / \mathrm{ml}$ for azoxystrobin. For C. fructicola isolates, the $\mathrm{EC}_{50}$ value was $0.036 \pm 0.031 \mu \mathrm{g} / \mathrm{ml}$ for difenoconazole, $6.305 \pm 0.903 \mu \mathrm{g} / \mathrm{ml}$ for tebuconazole, $0.140 \pm$ $0.074 \mu \mathrm{g} / \mathrm{ml}$ for prochloraz, and $>100 \mu \mathrm{g} / \mathrm{ml}$ for azoxystrobin (Table 5). The values indicated that both $C$. siamense and $C$. fructicola isolates were quite sensitive to prochloraz, difenoconazole, and tebuconazole but not to azoxystrobin. $C$. siamense isolates were more sensitive to prochloraz than $C$. fructicola isolates $(\alpha=0.05)$. There were no significant differences in the $\mathrm{EC}_{50}$ values against difenoconazole and tebuconazole between the two species.

Strong correlations were obtained between difenoconazole and prochloraz for $C$. fructicola $(P<0.05)$ and between difenoconazole and tebuconazole for $C$. siamense $(P<0.05)$ (Table 6$)$.

\section{Discussion}

This study aimed to characterize and compare the Colletotrichum spp. associated with anthracnose of strawberry in China using morphological characteristics and multilocus phylogenetic analyses. A subset of 62 isolates represented three distinct taxa: $C$. siamense
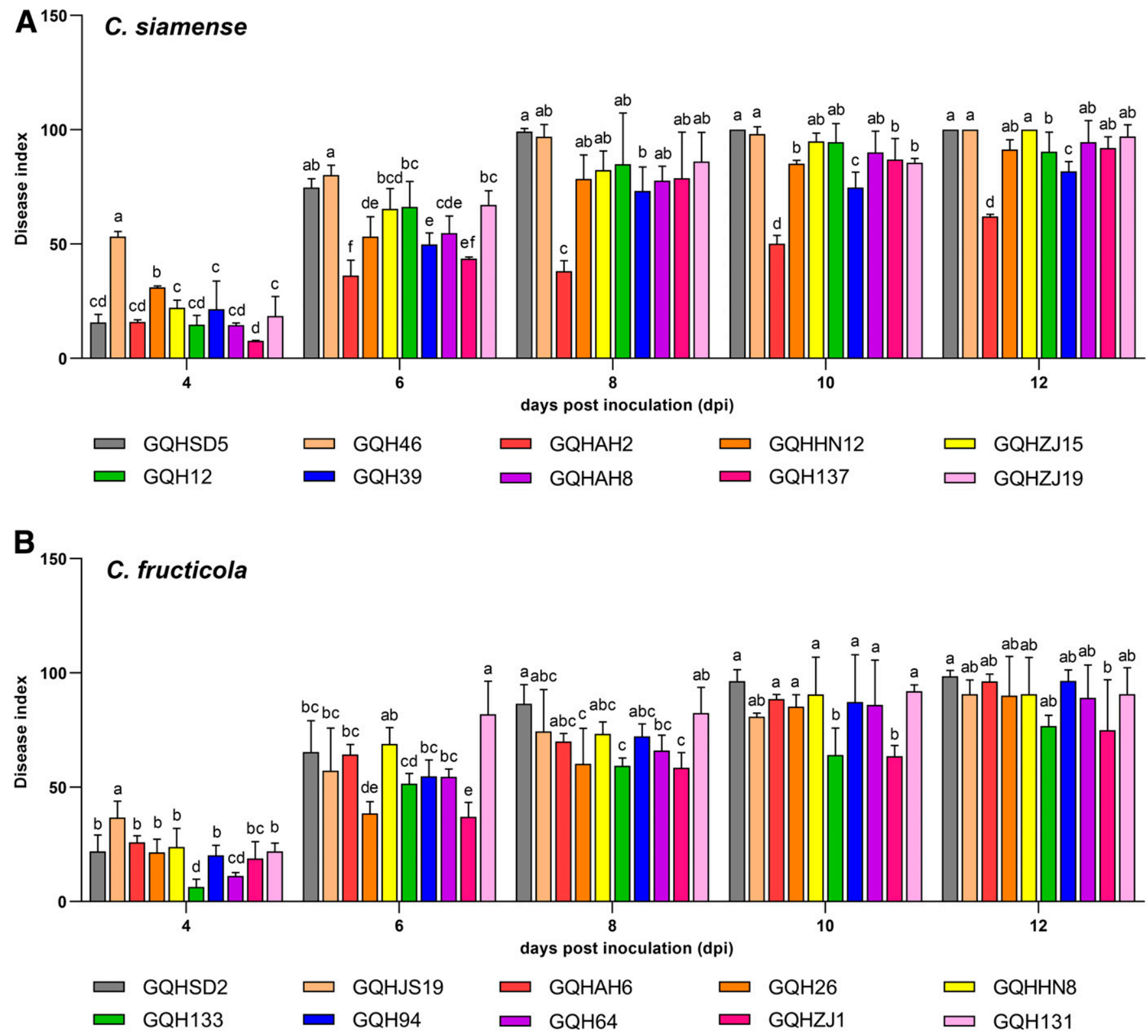

Fig. 5. Disease index for $\mathbf{A}$, Colletotrichum siamense $(n=10)$ and $\mathbf{B}, \mathbf{C}$. fructicola $(n=10)$ isolates on strawberry plants from eastern China. Mean scores of disease index. Mean values with the same letter do not differ significantly $(P=0.05)$ according to Duncan's multiple range test. 
(29 isolates, 46.8\%), C. fructicola (30 isolates, 48.4\%), and $C$. aenigma (3 isolates, $4.8 \%$ ). Their pathogenicity was confirmed in strawberry plants (leaves and petioles). The study also reported the fungicide sensitivities of Colletotrichum sp. complexes from strawberry in eastern China, which can optimize the selection of the most effective fungicides.

Both $C$. siamense and $C$. fructicola were originally described as causal agents of anthracnose on coffee berries in northern Thailand (Prihastuti et al. 2009). Different species in the $C$. gloeosporioides species complex are associated with strawberry anthracnose (Gan et al. 2017; Nam et al. 2013; Weir et al. 2012). Our results were in agreement with previous studies indicating that the $C$. gloeosporioides species complex is the most prevalent agent of strawberry anthracnose in China (Han et al. 2016; Jayawardena et al. 2016). Recently, Japanese strawberry cultivars have been the main cultivars grown in eastern China. C. siamense, $C$. fructicola, and C. aenigma are also the dominant fungal species causing strawberry anthracnose in Japan (Gan et al. 2017). We hypothesize that the Japanese strawberry cultivars are more susceptible to $C$. siamense, $C$. fructicola, and C. aenigma, resulting in the prevalence of strawberry anthracnose in both Japan and eastern China. C. acutatum and C. theobromicola (synonymous with $C$. fragariae, which is now in the $C$. gloeosporioides species complex) infect strawberry plants (Brooks 1931; Buddie et al. 1999) but no isolates of these species were identified in this study.

Temperature is a major factor affecting plant disease epidemics (Bonde et al. 2012; Wilson et al. 1990). Leandro et al. (2003) found that the optimum temperature ranges for $C$. acutatum conidial germination and appressorial development were 23.0 to 27.7 and 17.6 to $26.5^{\circ} \mathrm{C}$, respectively (Leandro et al. 2003). The temperature required for the optimal disease development of anthracnose on lentils ranged from 20 to $24^{\circ} \mathrm{C}$, with the greatest damage occurring at $28^{\circ} \mathrm{C}$ (Chongo and Bernier 2000). In recent years, Colletotrichum spp. have been increasingly prevalent in several cities of China that have hot and rainy summers. The effects of temperature on the growth rate of Colletotrichum spp. in vitro was evaluated in this study. Although there were no significant differences observed among the minimum and optimum growth temperatures of the three Colletotrichum spp. examined, the maximum growth temperatures were different (Fig. 4). C. siamense showed a stronger pathogenicity to strawberry plants and more tolerance to high temperatures than $C$. fructicola and $C$. aenigma, suggesting the potential threat of $C$. siamense, especially in areas with hot and rainy weather such as cities in eastern China.

C. siamense, C. fructicola, and C. aenigma were all pathogenic to leaves and petioles of strawberry plants. The species $C$. fructicola and $C$. siamense have broad host ranges and are recognized pathogens of chili, peach, pear, blueberry, and apple (De Silva et al. 2017; Ivic et al. 2013; Munir et al. 2016). The assessed pathogenicity levels of $C$. siamense and $C$. fructicola isolates were consistent with those of previous reports, indicating that $C$. siamense was more aggressive on strawberry than $C$. fructicola. $C$. aenigma isolates infect strawberry fruit and cause anthracnose disease symptoms (Baroncelli et al. 2015). Isolates of $C$. fructicola showed stronger pathogenicity levels than C. aenigma on both leaves and petioles of strawberry in Wuhan, China (Han et al. 2016), and isolates of C. aenigma-infected leaves and petioles had lower mean infection incidences than $C$. siamense and $C$. fructicola. These data, together with our results, indicate that, in comparison with $C$. siamense and $C$. fructicola, $C$. aenigma was probably not the dominant pathogen causing strawberry anthracnose in China.

More importantly, 10 isolates each of $C$. siamense and $C$. fructicola were selected for further pathogenicity testing. Most $C$. siamense and C. fructicola isolates shared a similar disease development process yet some individual isolates caused significantly greater or lower disease indices than other isolates. In particular, the disease index of GQHSD2 from $C$. fructicola was greater than that of GQHAH2 from $C$. siamense, although $C$. fructicola resulted in a lower disease severity than $C$. siamense $(P<0.05)$. Previous studies selected only a few isolates of Colletotrichum spp. isolated from different hosts for pathogenicity testing (Niu et al. 2016; Sharma and Shenoy 2014), and the variation in pathogenicity among isolates of different Colletotrichum spp. has rarely been reported. Gan et al. (2017) classified 115 isolates collected from strawberry plants in Japan into four species of the C. gloeosporioides species complex. In addition, although $C$. fructicola was identified as the predominant virulent species in Japan, both virulent and less virulent isolates of $C$. fructicola and $C$. siamense were also identified. This result indicated that specific species were not strictly correlated with virulence. These findings, together with the results in our study, imply that pathogenicity testing is required to select a large number of isolates to ensure the accuracy of the results and avoid a bias.

The application of agrochemicals is still an essential strategy for controlling strawberry anthracnose in China. Since the 1990s, benzimidazoles, including carbendazim and thiophanate-methyl, have been the main fungicide group extensively used for the control of strawberry anthracnose in China. QoI fungicides are commonly used to control anthracnose in many countries, including China (Baggio et al. 2018; Forcelini et al. 2016; Hu et al. 2015; Ji et al. 2014; Kongtragoul et al. 2020; Li et al. 2005; Yokosawa et al. 2017). Azoxystrobin is a QoI fungicide that was registered for strawberry in China in 2015 (China Pesticide Information Network; http://www.icama.org.cn/). The recommended concentration for strawberry anthracnose was approximately $500 \mu \mathrm{g} / \mathrm{ml}$. Furthermore, the resistance of the $C$. gloeosporioides species complex against QoI (azoxystrobin) fungicides has also been reported in various crops from many countries, such as $C$. gloeosporioides from strawberry in Japan (Inada et al. 2008), C. siamense from peach and blueberry in South Carolina in the United States (Hu et al. 2015), C. gloeosporioides from boxthorn and apple in Korea (Kim et al. 2016), and C. fructicola from apple in Japan (Yokosawa et al. 2017). Here, the $C$. siamense and $C$. fructicola isolates were all resistant to QoI (azoxystrobin) fungicides, indicating that these fungicides may not be suitable for the control of strawberry anthracnose, at least not in eastern China. The resistance could be addressed in the following ways: (i) $C$. fructicola has a teleomorph

Table 5. Summary of the sensitivity to four fungicides for Colletotrichum siamense and C. fructicola from strawberry plants in eastern Chinaw

\begin{tabular}{|c|c|c|c|c|c|c|c|}
\hline \multirow[b]{3}{*}{ FRAC $^{x}$} & \multirow[b]{3}{*}{ Chemical } & \multicolumn{6}{|c|}{$\mathrm{EC}_{50}(\mu \mathrm{g} / \mathrm{ml})$} \\
\hline & & \multicolumn{3}{|c|}{ C. siamense } & \multicolumn{3}{|c|}{ C. fructicola } \\
\hline & & $N^{\mathrm{y}}$ & Range & $\operatorname{Mean}^{z}$ & $N^{\mathbf{y}}$ & Range & $\operatorname{Mean}^{z}$ \\
\hline 11 & Azoxystrobin & 15 & $216.153-768.342$ & $>100$ & 15 & $100.968-745.629$ & $>100$ \\
\hline 3 & Difenoconazole & 15 & $0.009-0.096$ & $0.056 \mathrm{D} \pm 0.030$ & 15 & $0.003-0.089$ & $0.036 \mathrm{D} \pm 0.031$ \\
\hline 3 & Tebuconazole & 15 & $1.259-16.661$ & $8.885 \mathrm{~B} \pm 0.532$ & 15 & $0.853-12.950$ & $6.305 \mathrm{~B} \pm 0.903$ \\
\hline 3 & Prochloraz & 15 & $0.011-0.358$ & $0.122 * \mathrm{C} \pm 0.070$ & 15 & $0.017-0.541$ & $0.140 * \mathrm{C} \pm 0.074$ \\
\hline
\end{tabular}

${ }^{\mathrm{w}}$ Data were transformed using a $\log 10$ transformation before statistical analysis. $\mathrm{EC}_{50}=$ the fungicide concentration at which fungal development is inhibited by $50 \%$.

${ }^{x}$ Fungicide Resistance Action Committee (FRAC) code.

y Number of isolates.

${ }^{\mathrm{z}}$ Means followed by an asterisk (*) are significantly different between different species based on the two-tailed $t$ tests of transformed data with $\alpha=0.05$. Means followed by the same letter are not significantly different between fungicides within each species, based on the least significant difference tests of transformed data with $\alpha=0.05$. 
and mandatory sexual recombination that increases the risk of fungicide resistance development (Yokosawa et al. 2017) and (ii) point mutations in the mitochondrial cytochrome $b$ gene confer high levels of resistance to QoI fungicides (Ma and Michailides 2005). However, DMI fungicides are potential alternatives to benzimidazole fungicides for the management of strawberry anthracnose (Xu et al. 2014). In this study, the sensitivity levels of three DMI fungicides (difenoconazole, tebuconazole, and prochloraz) were determined. The sensitivities of $C$. siamense and $C$. fructicola to difenoconazole and prochloraz were consistently high. Members of the C. gloeosporioides species complex isolated from rubber trees in Hainan are more sensitive to prochloraz than to difenoconazole (Cao et al. 2017). Our results indicate that the C. gloeosporioides species complex isolated from strawberry plants was most sensitive to difenoconazole, less sensitive to prochloraz, and least sensitive to tebuconazole. This discrepancy indicates that variations in sensitivity to fungicides may be the result of different hosts and regions. The present study confirmed that the isolates of $C$. siamense were more sensitive to prochloraz than $C$. fructicola. The mean $\mathrm{EC}_{50}$ value for prochloraz in all of the tested $C$. siamense isolates in the present study was $0.122 \mu \mathrm{g} / \mathrm{ml}$. The isolates of $C$. fructicola were more sensitive to difenoconazole and tebuconazole than $C$. siamense isolates. In all the tested $C$. fructicola isolates in the present study, the mean $\mathrm{EC}_{50}$ values for difenoconazole and tebuconazole were $0.036 \mu \mathrm{g} / \mathrm{ml}$ and $6.305 \mu \mathrm{g} / \mathrm{ml}$, respectively. The sensitivities of these two species to difenoconazole, tebuconazole, and prochloraz were significantly different, revealing the importance of understanding their relative prevalence in a given region. Thus, more field studies are needed in the future.

In the present study, for $C$. fructicola only, there were correlations in the sensitivity levels between difenoconazole and prochloraz. For C. siamense, strong correlations were obtained between difenoconazole and tebuconazole. These may be related to DMI resistance. A positive cross-resistance was observed between tebuconazole and difenoconazole on the $C$. gloeosporioides species complex isolates from strawberry and grape plants. The DMI resistance resulted from multiple mechanisms such as mutations in the CYP51 gene that lead to the reduced affinity of DMI for the sterol $14 \alpha$-demethylase enzyme encoded by the CYP51 gene (Leroux and Walker 2011; Sang et al. 2015).

This study identified three Colletotrichum spp. (C. siamense, $C$. fructicola, and C. aenigma) from the $C$. gloeosporioides species complex affecting strawberry plants in six provinces of eastern China. C. siamense and C. fructicola were identified as the predominant virulent species. The fungicide sensitivity test showed that QoI fungicides (azoxystrobin) were not suitable for strawberry anthracnose control in eastern China. Instead, two DMI fungicides, difenoconazole and tebuconazole, could be used as alternatives. This provides insights into fungicide application strategies for the effective control of the disease. Further work is needed to analyze the influences of environmental variables and to conduct field trials to verify the effects of DMI fungicides. This may increase the ability to successfully manage strawberry anthracnose.

Table 6. Cross-sensitivity between azoxystrobin, difenoconazole, and tebuconazole inhibitor fungicides for isolates of Colletotrichum siamense and C. fructicola from strawberry plants in eastern China $^{\mathrm{z}}$

\begin{tabular}{lcccc}
\hline Chemical name & Azoxystrobin & Difenoconazole & Tebuconazole & Prochloraz \\
\hline Azoxystrobin & $\ldots$ & 0.21 & 0.20 & 0.01 \\
Difenoconazole & 0.04 & $\ldots$ & 0.28 & $0.60^{*}$ \\
Tebuconazole & 0.38 & $0.67^{*}$ & $\ldots$ & 0.34 \\
Prochloraz & 0.11 & 0.24 & 0.31 & $\ldots$ \\
\hline
\end{tabular}

$\mathrm{z}$ The correlation coefficient $(r)$ for the $C$. fructicola species complex $(n=15)$ is presented above the ellipses (...), whereas the $C$. siamense species complex $(n=15)$ is presented below the ellipses. The $r$ was determined via a comparison of $\log 50 \%$ effective concentration values for each fungicide from the individual species complex; an asterisk (*) indicates that the $r$ is significant at $P=0.05$.

\section{Acknowledgments}

We thank L. Benyon from Liwen Bianji, Edanz Group, China, for editing the English text of a draft of this manuscript.

\section{Literature Cited}

Anciro, A., Mangandi, J., Verma, S., Peres, N., Whitaker, V. M., and Lee, S. 2018. FaRCg1: A quantitative trait locus conferring resistance to Colletotrichum crown rot caused by Colletotrichum gloeosporioides in octoploid strawberry. Theor. Appl. Genet. 131:2167-2177.

Baggio, J. S., Wang, N.-Y., Peres, N. A., and Amorim, L. 2018. Baseline sensitivity of Colletotrichum acutatum isolates from Brazilian strawberry fields to azoxystrobin, difenoconazole, and thiophanate-methyl. Trop. Plant Pathol. 43:533-542.

Baroncelli, R., Zapparata, A., Sarrocco, S., Sukno, S. A., Lane, C. R., Thon, M. R., Vannacci, G., Holub, E., and Sreenivasaprasad, S. 2015. Molecular diversity of anthracnose pathogen populations associated with UK strawberry production suggests multiple introductions of three different Colletotrichum species. PLoS One 10:e0129140.

Bonde, M. R., Nester, S. E., and Berner, D. K. 2012. Effects of daily temperature highs on development of Phakopsora pachyrhizi on soybean. Phytopathology 102:761-768.

Brannen, P., and Smith, P. 2015. Southeast Regional Strawberry Integrated Pest Management Guide. North Carolina State University. https://strawberries.ces.ncsu.edu/ wp-content/uploads/2014/10/2015StrawberryIPMGuide1.pdf?fwd=no

Brooks, A. 1931. Anthracnose of strawberry caused by Colletotrichum fragariae, n. sp. Phytopathology 21:739-744.

Buddie, A. G., Martinez-Culebras, P., Bridge, P. D., Garcia, M. D., Querol, A., Cannon, P. F., and Monte, E. 1999. Molecular characterization of Colletotrichum strains derived from strawberry. Mycol. Res. 103:385-394.

Cai, L., Giraud, T., Zhang, N., Begerow, D., Cai, G., and Shivas, R. G. 2011. The evolution of species concepts and species recognition criteria in plan pathogenic fungi. Fungal Divers. 50:121-133.

Cai, L., Hyde, K. D., Taylor, P. W. J., Weir, B. S., Waller, J. M., Abang, M. M., Zhang, J. Z., Yang, Y. L., Phoulivong, S., and Liu, Z. Y., Prihastuti, H., Shivas, R. G., McKenzie, E. H. C., and Johnston, P. R. 2009. A polyphasic approach for studying Colletotrichum. Fungal Divers. 39:183-204.

Cao, X., Xu, X., Che, H., West, J. S., and Luo, D. 2017. Distribution and fungicide sensitivity of Colletotrichum species complexes from rubber tree in Hainan, China. Plant Dis. 101:1774-1780.

Cao, X., Xu, X., Che, H., West, J. S., and Luo, D. 2019. Three Colletotrichum species, including a new species, are associated to leaf anthracnose of rubber tree in Hainan, China. Plant Dis. 103:117-124.

Carbone, I., and Kohn, L. M. 1999. A method for designing primer sets for speciation studies in filamentous ascomycetes. Mycologia 91:553-556.

Chongo, G., and Bernier, C. C. 2000. Effects of host, inoculum concentration, wetness duration, growth stage, and temperature on anthracnose of lentil. Plant Dis. 84:544-548.

Damm, U., Cannon, P. F., Woudenberg, J. H., and Crous, P. W. 2012. The Colletotrichum acutatum species complex. Stud. Mycol. 73:37-113.

De Silva, D. D., Ades, P. K., Crous, P. W., and Taylor, P. W. J. 2017. Colletotrichum species associated with chili anthracnose in Australia. Plant Pathol. 66:254-267.

Denoyes-Rothan, B., Guérin, G., Délye, C., Smith, B., Minz, D., Maymon, M., and Freeman, S. 2003. Genetic diversity and pathogenic variability among isolates of Colletotrichum species from strawberry. Phytopathology 93 219-228.

FAOSTAT. 2016. Food and Agriculture Organization of the United Nations Cropping database. http://www.fao.org/faostat/en/\#home

Forcelini, B. B., Seijo, T. E., Amiri, A., and Peres, N. A. 2016. Resistance in strawberry isolates of Colletotrichum acutatum from Florida to quinoneoutside inhibitor fungicides. Plant Dis. 100:2050-2056.

Freeman, S., Horowitz, S., and Sharon, A. 2001. Pathogenic and nonpathogenic lifestyles in Colletotrichum acutatum from strawberry and other plants. Phytopathology 91:986-992.

Freeman, S., and Katan, T. 1997. Identification of Colletotrichum species responsible for anthracnose and root necrosis of strawberry in Israel. Phytopathology 87:516-521.

Gan, P., Nakata, N., Suzuki, T., and Shirasu, K. 2017. Markers to differentiate species of anthracnose fungi identify Colletotrichum fructicola as the predominant virulent species in strawberry plants in Chiba Prefecture of Japan. J. Gen. Plant Pathol. 83:14-22.

Glass, N. L., and Donaldson, G. C. 1995. Development of primer sets designed for use with the PCR to amplify conserved genes from filamentous ascomycetes. Appl. Environ. Microbiol. 61:1323-1330.

Hall, T. A. 1999. BioEdit: A user-friendly biological sequence alignment editor and analysis program for Windows 95/98/NT. Nucleic Acids Symp. Ser. 41:95-98.

Han, G., Li, Q., Sun, F., and Li, H. 2009. Identification of pathogen caused strawberry anthracnose and their resistance to carbendazim and diethofencarb. Zhejiang Agric. Sci. 6:1169-1172.

Han, Y. C., Zeng, X. G., Xiang, F. Y., Ren, L., Chen, F. Y., and Gu, Y. C. 2016 Distribution and characteristics of Colletotrichum spp. associated with anthracnose of strawberry in Hubei, China. Plant Dis. 100:996-1006. 
Horiuchi, S., and Hori, M. 1980. A simple greenhouse technique for obtaining high levels of clubroot incidence. Pages 33-35 in: Bull. Chugoku Natl. Agric. Exp. Stn. E.

Howard, C. M., Maas, J. L., Chandler, C. K., and Albregts, E. E. 1992. Anthracnose of strawberry caused by the Colletotrichum complex in Florida. Plant Dis. 76:976-981.

Hu, M. J., Grabke, A., Dowling, M. E., Holstein, H. J., and Schnabel, G. 2015. Resistance in Colletotrichum siamense from peach and blueberry to thiophanate-methyl and azoxystrobin. Plant Dis. 99:806-814.

$\mathrm{Hu}, \mathrm{S} .1990$. The preliminary investigation of strawberry diseases. Pages 58-59 in: Collect. Abstr. Fifth Meet. East China, Chin. Soc. Plant Pathol. Jiangsu.

Inada, M., Ishii, H., Chung, W., Yamada, T., Yamaguchi, J., and Furuta, A. 2008. Occurrence of strobilurin-resistant strains of Colletotrichum gloeosporioides (Glomerella cingulata), the causal fungus of strawberry anthracnose. Jpn. J. Phytopathol. 74:114-117.

Ivic, D., Voncina, D., Sever, Z., Simon, S., and Pejic, I. 2013. Identification of Colletotrichum species causing bitter rot of apple and pear in Croatia. J. Phytopathol. 161:284-286.

Jayawardena, R. S., Huang, J. K., Jin, B. C., Yan, J. Y., Li, X. H., Hyde, K. D., Bahkali, A. H., Yin, S. L., and Zhang, G. Z. 2016. An account of Colletotrichum species associated with strawberry anthracnose in China based on morphology and molecular data. Mycosphere 7:1147-1163.

Ji, M., Wu, X., Yao, K., Chen, H., Yang, J., Wang, L., Zhuang, Y. 2014. Identification of strawberry anthracnose pathogens and screening of germicides. Agric. Sci. Technol.. 1:94-98, 143.

Khoshnevisan, B., Rafiee, S., and Mousazadeh, H. 2013. Environmental impact assessment of open field and greenhouse strawberry production. Eur. J. Agron. 50:29-37.

Khoshnevisan, B., Rafiee, S., and Mousazadeh, H. 2014. Application of multilayer adaptive neuro-fuzzy inference system for estimation of greenhouse strawberry yield. Measurement 47:903-910.

Kim, S., Min, J., Back, D., Kim, H., Lee, S., and Kim, K. 2016. Assessment of QoI resistance in Colletotrichum spp. isolated from boxthorn and apple in Korea. (Abstr.) Phytopathology 106:S4.71

Kongtragoul, P., Imamoto, K., and Ishii, H. 2020. Resistance to quinone-outside inhibitor (QoI) fungicides in Colletotrichum species isolated from anthracnose disease occurring in Thailand. Curr. Appl. Sci. Technol. 20: 79-89.

Leandro, L. F. S., Gleason, M. L., Nutter, F. W., Jr., Wegulo, S. N., and Dixon, P. M. 2003. Influence of temperature and wetness duration on conidia and appressoria of Colletotrichum acutatum on symptomless strawberry leaves. Phytopathology 93:513-520.

Leroux, P., and Walker, A.-S. 2011. Multiple mechanisms account for resistance to sterol $14 \alpha$-demethylation inhibitors in field isolates of Mycosphaerella graminicola. Pest Manage. Sci. 67:44-59.

Li, H., Liu, Z., Wang, J., and Zhou, M. 2005. Baseline sensitivity of Colletotrichum gloeosporioides and $C$. capsici from capsium to azoxystrobin. Acta Phytopathol. Sin. 35:73-77.

Ma, Z., and Michailides, T. J. 2005. Advances in understanding molecular mechanisms of fungicide resistance and molecular detection of resistant genotypes in phytopathogenic fungi. Crop Prot. 24:853-863.

Marin-Felix, Y., Groenewald, J. Z., Cai, L., Chen, Q., Marincowitz, S., Barnes, I., Bensch, K., Braun, U., Camporesi, E., Damm, U., de Beer, Z. W., Dissanayake, A., Edwards, J., Giraldo, A., Hernandez-Restrepo, M., Hyde, K. D., Jayawardena, R. S., Lombard, L., Luangsa-Ard, J., McTaggart, A. R., Rossman, A. Y., Sandoval-Denis, M., Shen, M., Shivas, R. G., Tan, Y. P., van der Linde, E. J., Wingfield, M. J., Wood, A. R., Zhang, J. Q., Zhang, Y., and Crous, P. W. 2017. Genera of phytopathogenic fungi: GOPHY 1. Stud. Mycol. 86:99-216.

Munir, M., Amsden, B., Dixon, E., Vaillancourt, L., and Gauthier, N. A. W. 2016. Characterization of Colletotrichum species causing bitter rot of apple in Kentucky orchards. Plant Dis. 100:2194-2203.

Nam, M. H., Park, M. S., Lee, H. D., and Yu, S. H. 2013. Taxonomic re-evaluation of Colletotrichum gloeosporioides isolated from strawberry in Korea. Plant Pathol. J. 29:317-322.

Niu, X., Gao, H., Qi, J., Chen, M., Tao, A., Xu, J., Dai, Z., and Su, J. 2016. Colletotrichum species associated with jute (Corchorus capsularis L.) anthracnose in southeastern China. Sci. Rep. 6:25179.

Nylander, J. 2004. MrModeltest Version 2. Program distributed by the author. Uppsala University, Uppsala, Sweden.
O’Donnell, K., and Cigelnik, E. 1997. Two divergent intragenomic rDNA ITS2 types within a monophyletic lineage of the fungus Fusarium are nonorthologous. Mol. Phylogenet. Evol. 7:103-116.

O'Donnell, K., Nirenberg, H. I., Aoki, T., and Cigelnik, E. 2000. A multigene phylogeny of the Gibberella fujikuroi species complex: Detection of additional phylogenetically distinct species. Mycoscience 41:61-78.

Prihastuti, H., Cai, L., Chen, H., McKenzie, E. H. C., and Hyde, K. D. 2009 Characterization of Colletotrichum species associated with coffee berries in northern Thailand. Fungal Divers. 39:89-109.

Ronquist, F., Teslenko, M., van der Mark, P., Ayres, D. L., Darling, A., Höhna, S., Larget, B., Liu, L., Suchard, M. A., and Huelsenbeck, J. P. 2012. MrBayes 3.2: Efficient Bayesian phylogenetic inference and model choice across a large model space. Syst. Biol. 61:539-542.

Sang, H., Hulvey, J., Popko, J. T., Jr., Lopes, J., Swaminathan, A., Chang, T., and Jung, G. 2015. A pleiotropic drug resistance transporter is involved in reduced sensitivity to multiple fungicide classes in Sclerotinia homoeocarpa (F. T Bennett) . Mol. Plant Pathol. 16:251-261.

Sharma, G., and Shenoy, B. D. 2014. Colletotrichum fructicola and C. siamense are involved in chilli anthracnose in India. Arch. Phytopathol. Plant Prot. 47: 1179-1194.

Silva, D. N., Talhinhas, P., Várzea, V., Cai, L., Paulo, O. S., and Batista, D. 2012 Application of the Apn2/MAT locus to improve the systematics of the Colletotrichum gloeosporioides complex: An example from coffee (Coffea spp.) hosts. Plant Pathol. 104:396-409.

Suzuki, T., Tanaka-Miwa, C., Ebihara, Y., Ito, Y., and Uematsu, S. 2010. Genetic polymorphism and virulence of Colletotrichum gloeosporioides isolated from strawberry (Fragaria $\times$ ananassa Duchesne). J. Gen. Plant Pathol. 76:247-253.

Templeton, M. D., Rikkerink, E. H. A., Solon, S. L., and Crowhurst, R. N. 1992. Cloning and molecular characterization of the glyceraldehyde-3-phosphate dehydrogenase-encoding gene and cDNA from the plant pathogenic fungus Glomerella cingulata. Gene 122:225-230.

Thompson, J. D., Gibson, T. J., and Higgins, D. G. 2003. Multiple sequence alignment using ClustalW and ClustalX. Curr. Protoc. Bioinf. 00:2.3.1-2.3.22.

Udayanga, D., Manamgoda, D. S., Liu, X., Chukeatirote, E., and Hyde, K. D 2013. What are the common anthracnose pathogens of tropical fruits? Fungal Divers. 61:165-179.

Ureña-Padilla, A. R., MacKenzie, S. J., Bowen, B. W., and Legard, D. E. 2002 Etiology and population genetics of Colletotrichum spp. causing crown and fruit rot of strawberry. Phytopathology 92:1245-1252.

Van Hemelrijck, W., Debode, J., Heungens, K., Maes, M., and Creemers, P. 2010 Phenotypic and genetic characterization of Colletotrichum isolates from Belgian strawberry fields. Plant Pathol. 59:853-861.

Wang, Q., Zhang, L., Zhang, J., Shen, Q., Ran, W., and Huang, Q. 2012. Effects of compost on the chemical composition of SOM in density and aggregate fractions from rice-wheat cropping systems as shown by solid-state 13CNMR spectroscopy. J. Plant Nutr. Soil Sci. 175:920-930.

Weir, B. S., Johnston, P. R., and Damm, U. 2012. The Colletotrichum gloeosporioides species complex. Stud. Mycol. 73:115-180.

White, T., Bruns, T., Lee, S., and Taylor, J. 1990. Amplification and direct sequencing of fungal ribosomal RNA genes for phylogenetics. Pages 315-322 in: PCR Protocols: A Guide to Methods and Applications. M. A. Innis, D. H. Gelfand, J. J. Sninsky, and T. J. White, eds. Academic Press, Inc., San Diego, CA, U.S.A.

Wilson, L. L., Madden, L. V., and Ellis, M. A. 1990. Influence of temperature and wetness duration on infection of immature and mature strawberry fruit by Colletotrichum acutatum. Phytopathology 80:111-116.

Wong, F. P., and Midland, S. L. 2007. Sensitivity distributions of California populations of Colletotrichum cereale to the DMI fungicides propiconazole, myclobutanil, tebuconazole, and triadimefon. Plant Dis. 91:1547-1555.

Wong, F. P., and Wilcox, W. F. 2002. Sensitivity to azoxystrobin among isolates of Uncinula necator: Baseline distribution and relationship to myclobutanil sensitivity. Plant Dis. 86:394-404.

Xu, X. F., Lin, T., Yuan, S. K., Dai, D. J., Shi, H. J., Zhang, C. Q., and Wang, H. D. 2014. Characterization of baseline sensitivity and resistance risk of Colletotrichum gloeosporioides complex isolates from strawberry and grape to two demethylation-inhibitor fungicides, prochloraz and tebuconazole. Australas. Plant Pathol. 43:605-613.

Yokosawa, S., Eguchi, N., Kondo, K.-i., and Sato, T. 2017. Phylogenetic relationship and fungicide sensitivity of members of the Colletotrichum gloeosporioides species complex from apple. J. Gen. Plant Pathol. 83:291-298. 